\title{
Atrial Fibrillation Spatiotemporal Complexity Is Affected by Pulmonary Vein Isolation
}

\author{
Marianna Meo ${ }^{1,2,3}$, Carole Dumas-Pomier ${ }^{4}$, Mélèze Hocini ${ }^{1,2,3,5}$, Michel Haïssaguerre ${ }^{1,2,3,5}$, Rémi \\ Dubois $^{1,2,3}$ \\ ${ }^{1}$ IHU Liryc, Electrophysiology and Heart Modeling Institute, FBU, Pessac-Bordeaux, France \\ ${ }^{2}$ Univ. Bordeaux, CRCTB, U1045, Bordeaux, France \\ ${ }^{3}$ INSERM, CRCTB, U1045, Bordeaux, France \\ ${ }^{4}$ CardioInsight, Medtronic, Minneapolis, MN, USA \\ ${ }^{5}$ Bordeaux University Hospital (CHU), Electrophysiology and Ablation Unit, Pessac, France
}

\begin{abstract}
Introduction Pulmonary vein isolation (PVI) is the cornerstone of atrial fibrillation $(A F)$ ablation. However, it is still unclear how AF complexity observed on body surface is affected by this intervention. This study aims to evaluate whether PVI has an impact on AF complexity as measured through principal component analysis (PCA) of body surface potential maps (BSPMs). Methods BSPMs were acquired with a 252-lead vest in 22 persistent $A F$ patients (20 male, $62 \pm 11$ years, maximum AF duration: $10 \pm 18$ months) before and after PVI. The atrial fibrillatory wave signal $(9 \pm 6 s)$ was divided in 0.5 -s segments, and AF complexity was assessed by the normalized amplitude norm $d_{\epsilon}$ and the cosine similarity $\cos \left(\alpha_{\boldsymbol{\epsilon}}\right)$ of the multilead error $\epsilon$ between the input signal at the frame $(s)$ and its PCA projection onto a $3 D$ subspace computed in the previous segment $(s-1)$. AF organization was also quantified by the nondipolar component index (NDI), i.e., the amount of energy non-preserved by the $3 D$ dipolar approximation of cardiac activity in the frame $(s)$. Results $A$ significant reduction in AF complexity was measured by all markers after PVI $\left(d_{\epsilon}\right.$ and $\cos \left(\alpha_{\epsilon}\right): p<0.01$; NDI: $\left.p<0.0001\right)$. Conclusions $A F$ complexity can be reliably measured by the proposed BSPM features and reflect the impact of PVI.
\end{abstract}

\section{Introduction}

Pulmonary veins (PVs) have been demonstrated to often play a critical role in the pathogenesis of atrial fibrillation (AF) [1]. Currently, PV isolation (PVI) is the cornerstone for catheter ablation (CA) of paroxysmal AF. While the effectiveness of PVI alone for persistent AF treatment is still under debate $[2,3]$, none of the alternative ablation protocols so far proposed [4-6] has convincingly contributed to improve its outcome $[7,8]$, and PVI is still a first-line strat- egy for AF ablation, either alone or in combination with additional lesions [9]. Multiple studies have demonstrated that the electrophysiological and anatomical properties of PVs create a proarrhythmogenic substrate [10], including larger size, thicker myocardial tissue, shorter refractory periods and slower conduction velocity [11]. Electrical isolation of PVs can help neutralizing arrhythmogenic sources, either focal or reentrant, and preventing their propagation to other atrial regions $[12,13]$. PVI significantly affects atrial substrate, whose activity appears less fractionated and slower than at baseline [14]. Changes in the dominant frequency are also detectable on the standard electrocardiogram in response to PVI [15], although with some variability across the leads. More generally, AF complexity quantified by principal component analysis (PCA) of body surface potential maps (BSPMs) correlates with CA duration and strategy and is predictive of its outcome [16]. This study aims to investigate to which extent PVI affects AF organization measured from BSPMs. Variations in signal features determined by PCA as in $[16,17]$ were linked to changes in AF complexity occurring during PVI. Our approach provides deeper insights into the efficacy and the relevance of this intervention to persistent $\mathrm{AF}$ treatment.

\section{Methods}

\subsection{AF electrophysiology study and CA protocol}

We enrolled 22 persistent AF patients (20 male, $62 \pm 11$ years old, maximum AF duration: $10 \pm 18$ months) referred for CA. Biatrial electroanatomical mapping was performed with a multipolar catheter (Pentaray or Lasso, Biosense Webster), and atrial electrograms were continuously recorded through a computer-based digital amplifier/recorder system (Labsystem Pro, Bard Electrophysiol- 
ogy). Cycle length (CL) was measured in the left atrial appendage (LAA) at baseline and monitored during the procedure to assess local CA impact in the LA. All patients first underwent circumferential PVI. If AF was not terminated by PVI, extra-PV regions identified through noninvasive phase mapping were targeted [6]. The procedural endpoint was AF termination, i.e., organization to atrial tachycardia (AT) or sinus rhythm (SR). Electrical cardioversion was performed if $\mathrm{CA}$ failed to terminate $\mathrm{AF}$.

\subsection{BSPM data format and preprocessing}

BSPMs were acquired with a 252-lead vest (ECVUE, Cardioinsight Technologies) at $1 \mathrm{kHz}$ before and after PVI. The multilead atrial fibrillatory wave (f-wave) signal $(9 \pm 6$ s) was processed as in [16], yielding a $L \times N$ matrix $\mathbf{Y}=$ $[\mathbf{y}(1) \ldots \mathbf{y}(N)] \in \Re^{L \times N}$, where $L=252$ is the number of BSPM leads, and $N$ is the number of samples. An example is shown in Figure 1.

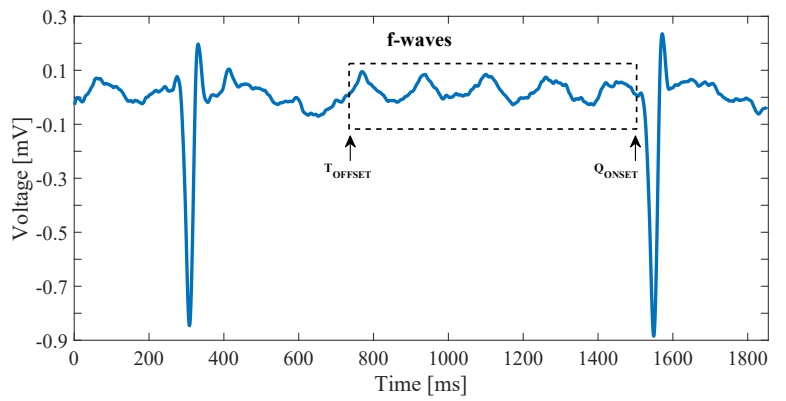

Figure 1. An example of f-wave signal segmented between the offset to the $\mathrm{T}$ wave ( $\mathrm{T}_{\text {OFFSET }}$ ) and the onset of the following $\mathrm{Q}$ wave (QONSET $)$ from a BSPM during AF.

\subsection{AF complexity features from BSPMs}

Multivariate measures of AF complexity were computed by PCA as in [17]. Body surface heart electrical activity can be modeled as a 3D dipole [18], and most of its energy can be well approximated by the 3 dominant PCA eigenvectors [19]. Accordingly, the input atrial signal $\mathbf{Y}$ was divided in 0.5-s frames, each projected on the 3D subspace $\mathbf{M}^{(s)}$ determined by PCA in the previous segment:

$$
\hat{\mathbf{Y}}^{(s+1)}=\mathbf{M}^{(s)}\left(\mathbf{M}^{(s)}\right)^{\#} \mathbf{Y}^{(s+1)}
$$

where $(\cdot)^{\#}$ is the MoorePenrose pseudoinverse operator. AF spatiotemporal organization was described in terms of the ability of the PCA components determined in the frame $(s)$ of the f-wave signal to be retrieved in $(s+1)$ : the lower their repetitiveness, the higher signal complexity. Thus, we assumed that the instantaneous PCA estimation error $\boldsymbol{\epsilon}(t)$ between the input signal $\mathbf{y}^{(s+1)}(t)$ at the frame $(s+1)$ and its PCA estimation $\hat{\mathbf{y}}^{(s+1)}(t)$ was linked to AF complexity and expressed in terms of the normalized magnitude norm:

$$
d_{\boldsymbol{\epsilon}}(t)=\frac{\left\|\hat{\mathbf{y}}^{(s+1)}(t)-\mathbf{y}^{(s+1)}(t)\right\|}{\left\|\mathbf{y}^{(s+1)}(t)\right\|},
$$

with higher $d_{\epsilon}$ values describing more disorganized AF, and cosine similarity, ranging from 0 to 1 (from low to high AF complexity):

$$
\cos (\boldsymbol{\epsilon})(t)=\frac{\left\langle\hat{\mathbf{y}}^{(s+1)}(t), \mathbf{y}^{(s+1)}(t)\right\rangle}{\left\|\hat{\mathbf{y}}^{(s+1)}(t)\right\|\left\|\mathbf{y}^{(s+1)}(t)\right\|},
$$

For the statistical analysis, the temporal average of these indices over each frame was considered. AF organization was also measured by the nondipolar component index (NDI), i.e., the energy retained by the PCA eigenvalues $\sigma_{\ell}$, $\ell=4, \ldots, L$ outside the subspace $\mathbf{M}^{(s)}$ at the frame $(s)$ :

$$
\mathrm{NDI}=1-\frac{\sum_{\ell=1}^{3} \sigma_{\ell}}{\sum_{\ell=1}^{\mathrm{L}} \sigma_{\ell}}
$$

with low NDI values associated with more organized AF patterns, which were accurately reconstructed by PCA.

\subsection{Statistical analysis}

The impact of PVI on body surface cardiac activity was assessed in terms of intraprocedural variations in the PCA markers of AF organization in the whole dataset. The same analysis was then performed in two subgroups of AF patients, i.e., those who were free from AF at the end of the entire procedure ("Termination") and those who did not experience AF termination by CA ("No termination"). To evaluate whether atrial substrate could be affected by PVI even in patients with more advanced AF forms, AF organization was also assessed before and after PVI both in persistent and long-lasting AF patients. Finally, we verified whether the location of the AF termination site in the left or the right atrium (LA vs RA) could be related to PVI efficacy as measured by PCA features.

Lilliefors test for normality was applied to all indices. A one-sample t-test was used to verify whether CL variations were significantly different from zero. Intergroup comparisons between BSPM features computed at a specific moment of the procedure (i.e., "Before PVI" or "After PVI") were made with an unpaired t-test for normally distributed data, or with a Mann Whytney test otherwise. Differences between pre- and post-PVI parameters were also tested for each group. Significance was taken for $p$-value $\leq 0.05$.

\section{Results}

\subsection{AF mapping and ablation}

As in [16], patients with AF duration of $<12$ months were assigned to the persistent AF group $(n=18)$, whereas 
3 patients were diagnosed with long-lasting $\mathrm{AF}(\geq 12$ months). AF duration was unknown in one patient. Baseline AF CL was $179 \pm 36 \mathrm{~ms}$. Mean ablation time was $61 \pm 25 \mathrm{~min}$ and $5 \pm 3$ atrial regions were targeted to terminate AF. AF was terminated in the PVs in 4 patients, in other LA sites in 2 patients, in the RA in 4 patients. AF was organized to AT in 4 patients, to SR in 6 patients.

\subsection{PVI and body surface AF complexity}

A significant reduction in AF complexity was measured by all PCA features after PVI (Figure 2). This evidence was corroborated by a significant prolongation of the initial AF CL ( $\Delta$ CL: $10 \pm 12 \mathrm{~ms}, p=0.002)$.
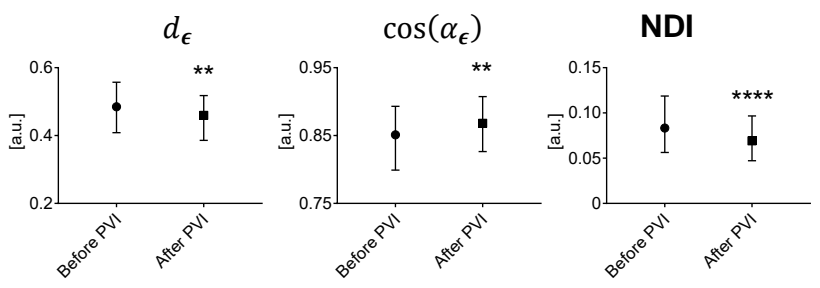

a.u.: arbitrary units; ${ }^{* * *}: p<0.0001 ;{ }^{* *}: p<0.01$

Figure 2. AF complexity and PVI $(n=22)$.

In persistent $\mathrm{AF}$ patients higher organization was measured by all indices after PVI, whereas in more severe cases changes in the atrial substrate due to this intervention could not significantly reflect on body surface (Figure 3). Surprisingly, BSPM complexity at baseline was higher in persistent rather than long-lasting AF patients, whereas it was comparable in both groups after PVI.

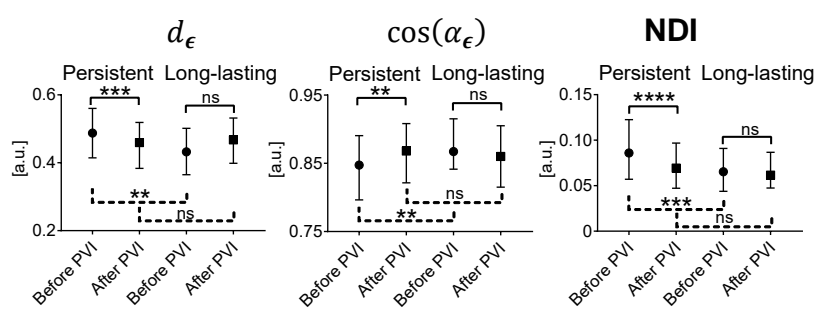

a.u.: arbitrary units; ns: not significant; ${ }^{\star \star}: p<0.01 ;{ }^{* \star *}: p<0.001 ;{ }^{* \star \star *}: p<0.0001$

Figure 3. AF complexity in persistent $(n=18)$ and longlasting $(n=3)$ patients before and after PVI.

AF complexity from BSPMs was lower after PVI regardless of CA outcome (Figure 4), with slower AF CL both in patients with ( $\Delta \mathrm{CL}: 14 \pm 16 \mathrm{~ms}, p=0.04)$ and without AF termination ( $\Delta \mathrm{CL}: 8 \pm 9 \mathrm{~ms}, p=0.02)$, and comparable in both groups $(p=0.3)$. Higher AF organization at baseline predicted $\mathrm{AF}$ termination by $\mathrm{CA}$, whereas there were no intergroup differences after PVI.

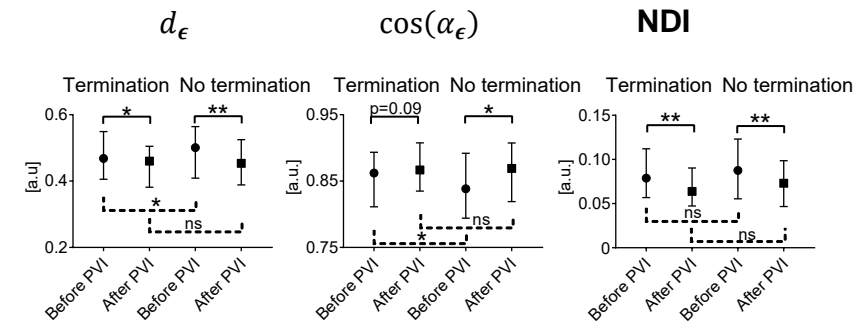

a.u.: arbitrary units; ns: not significant; *: $p<0.05 ;{ }^{* *}$ : $p<0.01$

Figure 4. AF complexity for effective $(n=10)$ and failed $(n=12)$ CA procedures before and after PVI.

There were no changes in BSPM complexity in patients with AF termination in the LA, whereas AF was more organized after PVI when the termination site was located in the RA (Figure 5). While all indices measured lower baseline AF complexity when the CA endpoint was achieved in the LA, after PVI they underlined higher AF organization in patients who were free from AF after RA ablation.

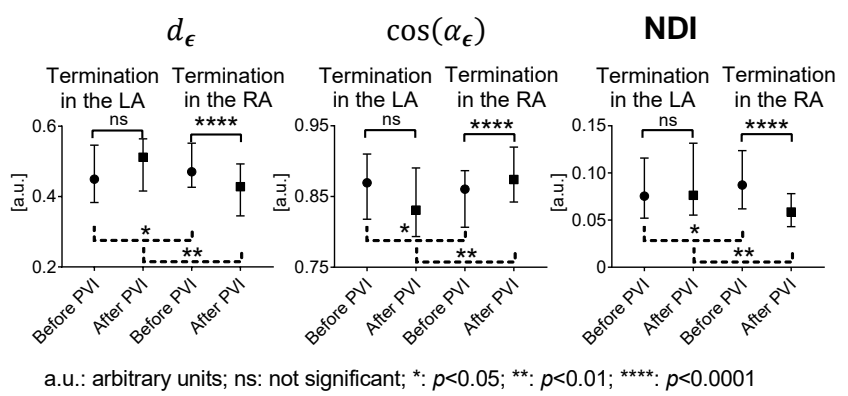

Figure 5. AF complexity for patients with AF termination in the LA $(n=6)$ and the RA $(n=4)$ before and after PVI.

\section{Discussion}

PVI significantly alters the atrial electrophysiology. Such modifications reflect on body surface as well, and they were effectively assessed by our PCA approach.

Electrical isolation of PVs substantially altered the atrial substrate in persistent AF patients, but not in the long-lasting ones, thus suggesting the presence of other drivers [20], which may be located at extra-PV sites and require a more extensive ablation [16].

As in $[15,16]$, more organized AF forms were more likely to be successfully treated by CA. Importantly, our method underlined how PVI contributed to modify the atrial substrate and simplify arrhythmia mechanisms, even when CA endpoint was not reached. These findings suggest that in some patients PVs may not only trigger AF, but also provide a substrate for its maintenance [21].

Our approach underlined higher disorganization at base- 
line in patients with AF termination in the RA, which hints at the implication of other drivers from this anatomical structure in AF maintenance [6]. In those subjects lower post-PVI BSPM complexity was measured, thus proving the major role of PVs in AF initiation and perpetuation. Albeit PVI contributed to organize LA activity (as confirmed by the prolongation of the LAA CL: $\triangle \mathrm{CL}: 26 \pm 11$ $\mathrm{ms}), \mathrm{AF}$ was still ongoing, partly due to other drivers in the RA, whose suppression was more crucial to reach the CA endpoint. By contrast, CL variations were more moderate when AF was organized in the LA ( $\triangle \mathrm{CL}: 6 \pm 14 \mathrm{~ms}$ ) rather than in the RA ( $p=0.04)$, thus confirming that PVs were less critical to treat AF than other LA sites.

\section{Conclusions}

This study showed that our PCA approach can quantify AF complexity and assess the impact of PVI, which plays a crucial role in AF ablation. However, our results confirm that sometimes PVI alone may not be sufficient to terminate $\mathrm{AF}$, and the underlying mechanisms should be clarified and validated by further intracardiac measures.

\section{Acknowledgements}

This study was funded by the French Government as part of the "Investments of the Future" program managed by the National Research Agency (ANR), grant reference ANR-10-663 IAHU-04. Dr Meo is funded by a LefoulonDelalande Foundation fellowship administered by the Institute of France.

\section{References}

[1] Haïssaguerre M, Jaïs P, Shah DC, et al. Spontaneous initiation of atrial fibrillation by ectopic beats originating in the pulmonary veins. N Engl J Med 1998;339(10):659-666.

[2] Chen S, Hsieh M, Tai $C$, et al. Initiation of atrial fibrillation by ectopic beats originating from the pulmonary veins: electrophysiological characteristics, pharmacological responses, and effects of radiofrequency ablation. Circulation 1999;100(18):1879-1886.

[3] Seitz J, Horvilleur J, Curel L, et al. Active or passive pulmonary vein in atrial fibrillation: is pulmonary vein isolation always essential? Heart Rhythm 2014;11(4):579-586.

[4] Nademanee K, Lockwood E, Oketani N, Gidney B. Catheter ablation of atrial fibrillation guided by complex fractionated atrial electrogram mapping of atrial fibrillation substrate. J Cardiol 2010;55(1):1-12.

[5] Narayan. SM, Krummen DE, Shivkumar K, et al. Treatment of atrial fibrillation by the ablation of localized sources: CONFIRM (Conventional Ablation for Atrial Fibrillation With or Without Focal Impulse and Rotor Modulation) trial. J Am Coll Cardiol 2012;60(7):628-636.

[6] Haïssaguerre M, Hocini M, Denis A, et al. Driver domains in persistent atrial fibrillation. Circulation 2014;130:530 538.

[7] Verma A, Jiang C, Betts TR, et al. Approaches to catheter ablation for persistent atrial fibrillation. N Engl J Med 2015; 372(19):1812-1822.

[8] Kirchhof P, Calkins H. Catheter ablation in patients with persistent atrial fibrillation. Eur Heart J 2017;38(1):20-26.

[9] Dagres N, Bongiorni MG, Larsen TB, et al. Current ablation techniques for persistent atrial fibrillation: results of the European Heart Rhythm Association Survey. Europace 2015;17(10):1596-1600.

[10] Mahida S, Sacher F, Derval N, et al. Science linking pulmonary veins and atrial fibrillation. Arrhythm Electrophysiol Rev 2015;4(1):40-43.

[11] Jaïs P, Hocini M, Macle L, et al. Distinctive electrophysiological properties of pulmonary veins in patients with atrial fibrillation. Circulation 2002;106(19):2479-2485.

[12] Hocini M, Sanders P, Jas P, et al. Prevalence of pulmonary vein disconnection after anatomical ablation for atrial fibrillation: consequences of wide atrial encircling of the pulmonary veins. European heart journal 2005;26(7):696-704.

[13] Shah D. Electrophysiological evaluation of pulmonary vein isolation. EP Europace 2009;11(11):1423-1433.

[14] Lin YJ, Tai CT, Kao T, et al. Spatiotemporal organization of the left atrial substrate after circumferential pulmonary vein isolation of atrial fibrillation. Circ Arrhythm Electrophysiol 2009;2(3):233-241.

[15] Lankveld T, Zeemering S, Scherr D, et al. Atrial fibrillation complexity parameters derived from surface ECGs predict procedural outcome and long-term follow-up of stepwise catheter ablation for atrial fibrillation. Circ Arrhythm Electrophysiol 2016;9(2):1-10.

[16] Meo M, Pambrun T, Derval N, et al. Noninvasive assessment of atrial fibrillation complexity in relation to ablation characteristics and outcome. Front Physiol 2018;9:929.

[17] Meo M, Potse M, Puyo S, et al. Non-invasive assessment of spatiotemporal organization of ventricular fibrillation through principal component analysis. Comput Cardiol 2017;44:1-4.

[18] Malmivuo J, Plonsey R. Principles and applications of bioelectric and biomagnetic fields. Oxford University Press., 1995.

[19] Lux RL, Evans AK, Burgess MJ, et al. Redundancy reduction for improved display and analysis of body surface potential maps. Circ Res 1981;49:186-196.

[20] Lim HS, Hocini M, Dubois R, et al. Complexity and distribution of driversinrelation to duration of persistentatrialfibrillation. J Am Coll Cardiol 2017;69(10):1257-1269.

[21] Haïssaguerre M, Sanders P, Hocini M, et al. Changes in atrial fibrillation cycle length and inducibility during catheter ablation and their relation to outcome. Circulation 2004;109:3007-3013.

Address for correspondence:

Marianna Meo

IHU Liryc Av. Hâut Lévêque 33604 Pessac-Bordeaux, France

marianna.meo@ihu-liryc.fr 